\title{
Sex chromosome and sex locus characterization in goldfish, Carassius auratus (Linnaeus, 1758)
}

\author{
Ming Wen ${ }^{1,2}$, Romain Feron 2,3,4, Qiaowei Pann ${ }^{2,3}$, Justine Guguin², Elodie Jouanno², Amaury Herpin², \\ Christophe Klopp ${ }^{5,6}$, Cedric Cabau ${ }^{6}$, Margot Zahm ${ }^{6}$, Hugues Parrinello ${ }^{7}$, Laurent Journot ${ }^{7}$, Shawn M. Burgess ${ }^{8}$, \\ Yoshihiro Omori ${ }^{9,10}$, John H. Postlethwait ${ }^{11}$, Manfred Schartl ${ }^{12,13}$ and Yann Guiguen ${ }^{2^{*}}$ (D)
}

\begin{abstract}
Background: Goldfish is an important model for various areas of research, including neural development and behavior and a species of significant importance in aquaculture, especially as an ornamental species. It has a male heterogametic $(X X / X Y)$ sex determination system that relies on both genetic and environmental factors, with high temperatures being able to produce female-to-male sex reversal. Little, however, is currently known on the molecular basis of genetic sex determination in this important cyprinid model. Here we used sequencing approaches to better characterize sex determination and sex-chromosomes in an experimental strain of goldfish.

Results: Our results confirmed that sex determination in goldfish is a mix of environmental and genetic factors and that its sex determination system is male heterogametic (XX/XY). Using reduced representation (RAD-seq) and whole genome (pool-seq) approaches, we characterized sex-linked polymorphisms and developed male specific genetic markers. These male specific markers were used to distinguish sex-reversed XX neomales from XY males and to demonstrate that $X X$ female-to-male sex reversal could even occur at a relatively low rearing temperature $\left(18^{\circ} \mathrm{C}\right)$, for which sex reversal has been previously shown to be close to zero. We also characterized a relatively large non-recombining region ( $11.7 \mathrm{Mb}$ ) on goldfish linkage group 22 (LG22) that contained a high-density of male-biased genetic polymorphisms. This large LG22 region harbors 373 genes, including a single candidate as a potential master sex gene, i.e., the anti-Mullerian hormone gene $(\mathrm{amh})$. However, no sex-linked polymorphisms were detected in the coding DNA sequence of the goldfish amh gene.
\end{abstract}

Conclusions: These results show that our goldfish strain has a relatively large sex locus on LG22, which is likely the Y chromosome of this experimental population. The presence of a few XX males even at low temperature also suggests that other environmental factors in addition to temperature could trigger female-to-male sex reversal. Finally, we also developed sex-linked genetic markers, which will be important tools for future research on sex determination in our experimental goldfish population. However, additional work would be needed to explore whether this sex locus is conserved in other populations of goldfish.

Keywords: Goldfish, RADseq, Poolseq, Sex determination, Sex markers, Male genome assembly

\footnotetext{
* Correspondence: yann.guiguen@inrae.fr

${ }^{2}$ INRAE, LPGP, 35000 Rennes, France

Full list of author information is available at the end of the article
}

C C The Author(s). 2020 Open Access This article is licensed under a Creative Commons Attribution 4.0 International License, which permits use, sharing, adaptation, distribution and reproduction in any medium or format, as long as you give appropriate credit to the original author(s) and the source, provide a link to the Creative Commons licence, and indicate if changes were made. The images or other third party material in this article are included in the article's Creative Commons licence, unless indicated otherwise in a credit line to the material. If material is not included in the article's Creative Commons licence and your intended use is not permitted by statutory regulation or exceeds the permitted use, you will need to obtain permission directly from the copyright holder. To view a copy of this licence, visit http://creativecommons.org/licenses/by/4.0/ The Creative Commons Public Domain Dedication waiver (http://creativecommons.org/publicdomain/zero/1.0/) applies to the data made available in this article, unless otherwise stated in a credit line to the data. 


\section{Background}

Goldfish, Carassius auratus (Linnaeus, 1758), is a domesticated fish species originating from central Asia and China that has been introduced throughout the world. Goldfish belongs to the Cyprinidae family and is considered as an important fish model for research in endocrinology [1, 2], developmental biology [3, 4] or fish pathology [5]. Thanks to the recent availability of a whole genome sequence assembly [6], goldfish is also now becoming a key model species for studies on genomics and cyprinid genome evolution. It is also a species of high aquaculture importance especially as an ornamental species, with many beautiful and sometimes bizarre phenotypes [7].

Unlike birds and mammals, sex determination in teleost is highly dynamic, with frequent turnovers of both sex determination (SD) systems [8] and master sex determining genes (MSD) $[9,10]$. Currently about half a dozen different master sex determining genes have been identified in teleosts, including $d m r t 1$ (doublesex and mab-3 related transcription factor 1) in the Japanese medaka, Oryzias latipes (Temminck and Schlegel 1846) [11], $s d Y$ (sexually dimorphic on the Y-chromosome) in rainbow trout [12], amh (anti-Mullerian hormone) in Northern pike, Nile tilapia and pejerrey [13-15], amhr2 (anti-Mullerian Hormone Receptor Type 2) in yellow perch and the Takifugu pufferfish [16, 17], gsdf (gonadal somatic cell derived factor) in sablefish and Luzon medaka, O. luzonensis, (Herre \& Ablan, 1934) [18, 19], gdf6a (growth differentiation factor 6a) in the turquoise killifish [20] and sox3 (SRY-box transcription factor 3) in the Indian ricefish O. dancena, (Hamilton, 1822) [21]. MSD turnover can be evolutionarily frequent as this has been shown for instance in various ricefish species, in which many MSD switches have been described within different species of the genus Oryzias [22]. In addition to genetic determinants, environmental factors -- especially temperature -- have also been shown to play a pivotal role in teleost sex determination [23].

Since the late 1960s, the goldfish sex determination system has been characterized as male heterogametic (XX/ XY) [24]. More recently, a strong temperature influence on sex-ratios has also been characterized in goldfish, with high rearing temperature treatments inducing complete masculinization of chromosomally all-female genotypes (XX neomales) when applied during early 3 months development [25]. The molecular mechanisms of genetic sex determination, however, are still unknown not only in goldfish, but also in any member of the Cyprinidae family.

Because of new high throughput sequencing technologies and the availability of a whole genome sequence assembly for goldfish [26], we implemented both reduced representation (i.e., Restriction-site associated DNA sequencing (RAD-seq) $[27,28]$, and whole genome (i.e., Pool sequencing (Pool-seq) $[29,30]$ ) approaches to identify sexlinked genetic polymorphisms in goldfish. We verified that identified sex-linked markers strictly segregated with the $\mathrm{Y}$ chromosome, and we characterized the extent of $\mathrm{Y}$ chromosome differentiation. Although our experiments did not identify a strong candidate sex-determining gene, these results lay a solid foundation for further molecular exploration of sex determination in our experimental goldfish population.

\section{Results}

Characterization of sex-linked $\mathrm{Y}$ chromosome markers in an experimental goldfish strain

Because goldfish sex determination is highly sensitive to temperature [1], with high temperature leading to the masculinization of some XX females producing XX neomales, we first searched for sex-linked markers using a RAD-seq approach that kept track of phenotypes and genotypes, potentially enabling the discrimination of XX neomales from XY genetic males. From our RAD-seq data, we identified 32 polymorphic/specific RAD-tags that were present in 12-15 males among the 30 phenotypic males used in this experiment, and completely absent in all the 30 phenotypic females (Fig. 1a, Additional file 1). These results suggest a male heterogametic genetic sex determination system (XX/XY) as previously shown in goldfish [24], but with a rather high occurrence of XX neomales (around 50\%) in this population of two-year old animals raised outdoor and obtained from different batches of animals with different spawning times i.e., from May-June to late September.

To validate the hypothesis that these markers were linked to the heterogametic sex (XY) and the Y chromosome, we first sequenced using Illumina reads and assembled a draft genome sequence of a male goldfish identified as a putative XY male based on the polymorphic/specific RAD-tags (see Material \& Methods) and blasted these 32 marker sequences against this genome assembly. This analysis returned 20 contigs with highly significant matches (Additional file 2) spanning a total of $0.24 \mathrm{Mb}$. By anchoring these sex-linked RAD sequences on our genome assembly, we were able to design three putative $\mathrm{Y}$-allele specific primer pairs that were used to genotype the same individual animals that were used for the RAD-seq analysis. PCR genotyping using these three primer pairs accurately discriminated putative XY genetic males from putative XX neomales and females (Fig. 1b, Additional file 5), validating that these primers accurately identified the two types of males found in our RAD-seq analysis. We then genotyped male breeders from our experimental stock with these primers and selected one putative XX neomale (breeder 1, negative PCR amplifications) and one putative XY male (breeder 2, positive PCR amplifications); and both individuals were crossed to the same $\mathrm{XX}$ female to generate two separate batches of fish. If our Y- 
A

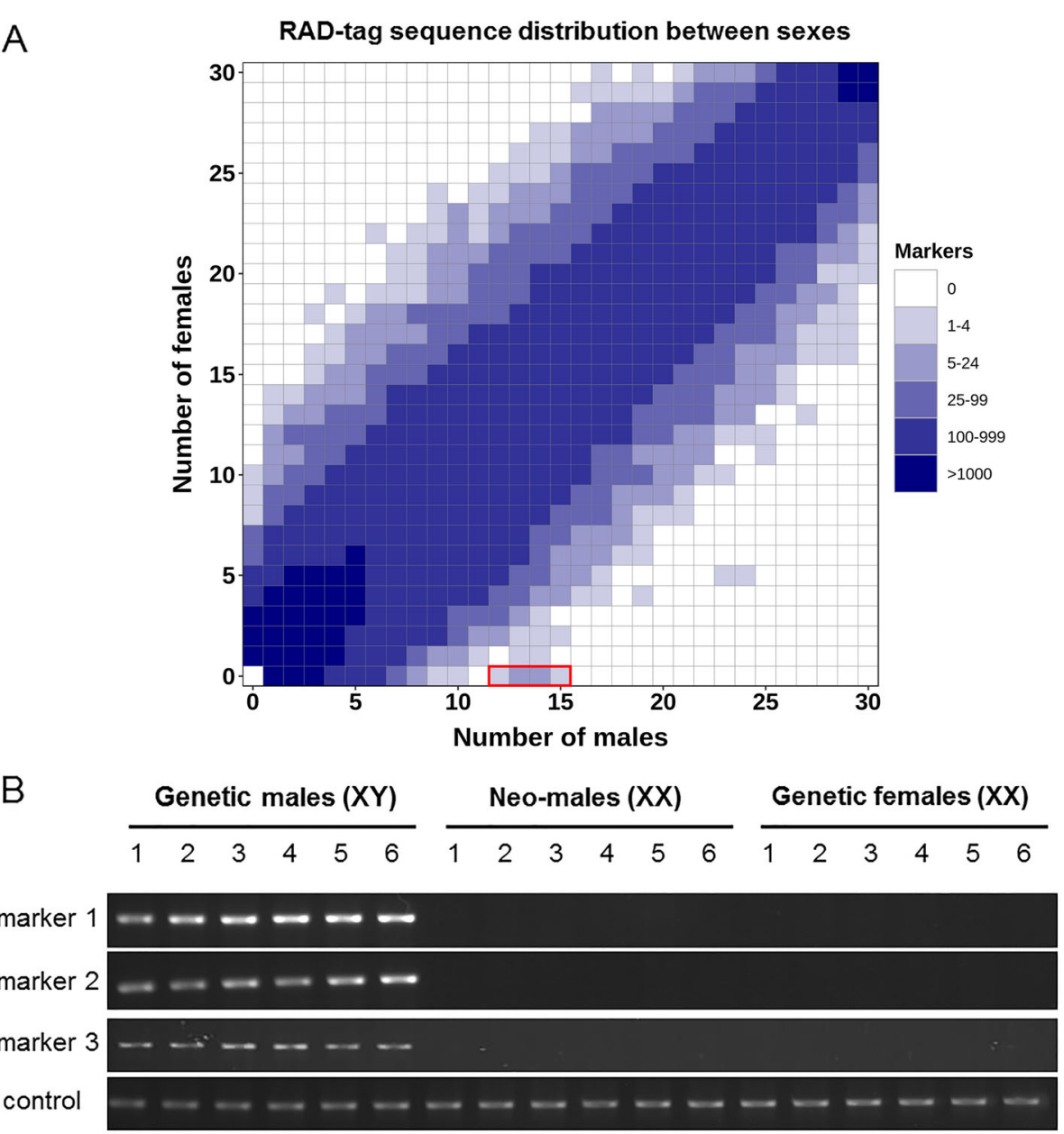

B

Fig. 1 RAD-sex tags and male-specific markers in goldfish. a Haplotypes heatmap in phenotypic males and females' goldfish. Each cell in the heatmap represents the number of haplotypes presented in $x$ phenotypic males and $y$ phenotypic females ( $x$ : cumulative number of males, $y$ : cumulative number of females). Haplotypes present in more than 12 males and absent in all females were identified as male-specific haplotypes (highlighted by red box). $\mathbf{b}$ Genotyping of goldfish males and females with three Y-allele primer pairs and one autosomal primer pair used as a positive control. Goldfish are categorized into three groups i.e., putative genetic males (XY), putative XX neomales, and genetic females by combining the results of both Y-allele genotyping and sex phenotyping. The original, unprocessed gel images of this figure are available in additional file 5

allele specific primers correctly identify the Y chromosome, then our putative $\mathrm{XX}$ neomale should give only female offspring and the putative $\mathrm{XY}$ male should give both male and female offspring. These two experimental populations were then reared at low temperature $\left(18^{\circ} \mathrm{C}\right)$ during the first 3 months after fertilization to minimize high temperature masculinization [25], and were subsequently maintained at $24^{\circ} \mathrm{C}$ for nine additional months before the identification of the phenotypic sex. Results from the histological examination of the offspring gonads of the putative XX neomale identified 7 fish with testes, 83 fish with ovaries, and 41 fish with undifferentiated gonads (Table 1). Gonadal histology of the offspring of the putative XY revealed 48 animals with testes, 65 with ovaries, and 14 with undifferentiated gonads (Table $1)$. The proportion of well characterized males and females in these two experimental populations (Table 1), suggests that male breeder 1 was an XX neomale with a well characterized female to male offspring ratio of 11.8 (83:7), indicative of a potential all-female population with a slight percentage of female-to-male sex-reversal, and that breeder 2 was a genetic XY male with a well characterized female to male offspring ratio of 1.3 (65: 48) indicative of a potential normal population with a 50:50 sex ratio. In agreement with these results, none of

Table 1 Phenotypic sex in two goldfish populations Population $\quad \mathrm{N}$. of male $\mathrm{N}$. of female $\mathrm{N}$. of fish with undetermined sex \begin{tabular}{llll}
\hline$P(X X)$ & 7 & 83 & 41
\end{tabular}

$\begin{array}{llll}P(X Y) & 48 & 65 & 14\end{array}$

$P(X X)$ putative neomale $(X X)$ offspring population; $P(X Y)$ putative genetic male $(\mathrm{XY})$ offspring population 
the XX neomale offspring produced a positive PCR amplification for our three $\mathrm{Y}$-allele specific primer pairs (Fig. S3, Table 2, Additional file 5), and all 48 phenotypic males but only one of 65 phenotypic female offspring from the XY phenotypic male produced positive amplifications (Fig. S4, Table 2, Additional file 5).

\section{Characterization of sex chromosome and sex-determining region (SDR)}

Using the three Y-allele specific primer pairs described above, we genotyped goldfish individuals and selected 30 phenotypic and genotypic males that were used along with 30 phenotypic females to contrast whole genome sex differences by pool-sequencing analysis [29]. Pool-sequencing reads from the respective XY male and phenotypic female pools were mapped to the high contiguity goldfish female genome assembly [2] to characterize genomic regions enriched for sex-biased signals, i.e., sex coverage differences or sex-biased Single Nucleotide Polymorphism (SNP) differences. Whole genome analysis of SNP distribution (Figs. 2 and 3a) revealed a strong sex-linked signal in males on linkage group 22 (LG22) and two unplaced scaffolds [National Center for Biotechnology Information Accession numbers: NW_020523543.1 (https://www.ncbi.nlm.nih. gov/nuccore/NW_020523543.1/) and NW_020523609.1 (https://www.ncbi.nlm.nih.gov/nuccore/NW_020523609.1)] with a high density of observed SNPs being heterozygous in the male pool and homozygous in the female pool (Y-specific allele). Interestingly, of the 32 markers found using the RAD-Seq approach, 7 tags were enriched in the unplaced scaffold NW_020523543.1 (Fig. 3c), confirming by a second approach that this scaffold is part of the SD locus in goldfish. These regions with a high density of male-specific SNPs (Fig. 3) are potential sex-determining regions that could contain the goldfish master sex determining gene. LG22, being the only linkage group with a large sex determining region (SDR, highlighted by a black box on Fig. 3a, c, d) containing a high-density of male-specific SNPs ( 11.7 Mb), likely corresponds to the $\mathrm{Y}$ sex chromosome of our goldfish population. However, it is important to note that this sex-specific signal on LG22 does not cover a single contiguous region, as it would be expected for such an SDR, but is instead broken in a few smaller regions with a high density of male-specific SNPs (Fig. 3a). This fragmented signal could be due to 1) quality issues of the reference

Table 2 Goldfish Y-allele sex-linkage

\begin{tabular}{lllll}
\hline Population & Male $^{\mathrm{a}}$ & Female $^{\mathrm{a}}$ & Undetermined sex $^{\mathrm{a}}$ & Sex linkage \\
\hline $\mathrm{P}(\mathrm{XX})$ & $0 / 7$ & $0 / 83$ & $0 / 41$ & NS \\
$\mathrm{P}(\mathrm{XY})$ & $48 / 48$ & $1 / 65$ & $10 / 14$ & $* * *$
\end{tabular}

${ }^{a} Y$-allele positive genotyping / total number of samples. $P(X X)$ : putative neomale $(X X)$ offspring population; $P(X Y)$ : putative genetic male $(X Y)$ offspring population; NS: Non-significant. Fisher's exact test was applied for statistics in $\mathrm{R}$ genome we have used [6] in our analysis, potentially because a wrong ordering and/or orientation of the contigs in the reference genome, 2) intra-populational rearrangements between the strain that has been sequenced and our goldfish population or 3) because of some large male-specific inversions on the $\mathrm{Y}$ compared to the $\mathrm{X}$ chromosome of this reference genome made from a gynogenetic XX female.

We also observed, however, some smaller signals with less dense sex-linked SNPs in other linkage groups (Fig. 2a) like for instance on LG47 (Fig. S1) with both male and female sex-linked signals. Interestingly, LG47 is paralogous to LG22 stemming from the Cyprinidae whole genome duplication [6]. Indeed, due to this recent common ancestry, these two chromosomes share large homologous and syntenic regions (Fig. S2) that could have resulted in some false remapping of the poolsequencing reads leading to some of these secondary minor signals.

\section{Identification of candidate SD genes}

Searches for annotated genes by BLAST within the 20 contigs found in our male goldfish draft genome assembly based on the RAD-Seq approach did not return any matches for a candidate SD gene, but mostly transposable elements (Additional file 3). In addition, all genes within the SDR $(N=373)$ from the high contiguity goldfish female genome assembly [2], were extracted because they are potential candidates for being SD gene(s) (Additional file 4). None of these genes were likely candidates as master sex determining genes based on their annotation with the exception of the anti-Mullerian hormone gene $(a m h)$ that was found in the SDR region at position $8,483,797-8,488,623$ bp on LG22 (Fig. 3b), as this gene has been reported to be a sex-determining gene in other fish species $[14,15]$. However, by looking at the remapping of the Pool-sequencing reads in the amh locus, we identified male-specific SNPS only in the non-coding regions of this gene, i.e., in the $3^{\prime}$ and $5^{\prime}$ untranslated regions, introns and the $5 \mathrm{~kb}$ promoter region, but we did not find any male-specific SNPs in the coding sequence of goldfish $a m h$. In the LG22 region with the highest density of sex-specific SNPs i.e., between 20.0 and $20.1 \mathrm{Mb}$, there are two annotated genes, Stromal cell-derived factor 1 $(s d f 1)$ and Xaa-Pro aminopeptidase 1-like (xpnpep1-like). Both are unlikely candidates as potential master sex determining genes.

\section{Discussion}

Though goldfish is an important economic ornamental fish and a useful model for studying development, evolution, neuroscience, and human disease [3], characterization of goldfish sex-specific sequences and potential sex chromosomes have not been reported. In this study, we explored goldfish sex determination using two complementary 

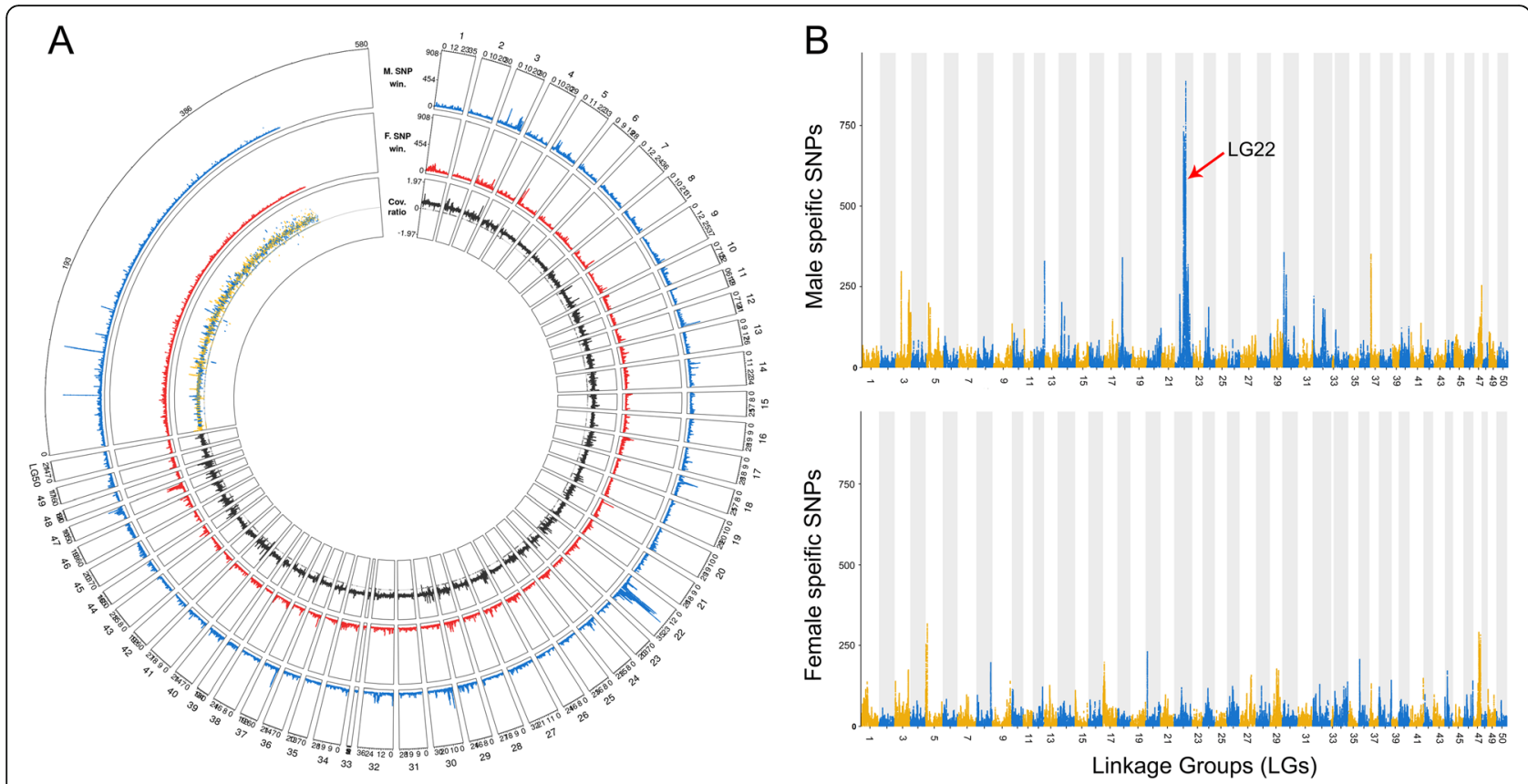

Fig. 2 Sex determining regions identified by remapping the Pool-seq male and female reads onto the female genome assembly. SNPs were counted using $100 \mathrm{~kb}$ sliding window with an output point every $500 \mathrm{bp}$. a Circular plot showing the genome wide metrics of the Pool-seq analysis. All the 50 goldfish linkage groups (LGs) are labelled with their LG number and all unplaced scaffolds are fused together. Outer to inner tracks show respectively: the male-specific SNPs, the female-specific SNPs, and the reads depth ratio between males and females. b Manhattan plot of the male- and female-specific SNPs showing a strong enrichment of male-specific SNPs on LG22

whole-genome approaches and found that this species has a $\mathrm{XX} / \mathrm{XY}$ sex determination system as previously described [24] and a large, non-recombining sex determination region on LG22. Although RAD-sequencing or pool-sequencing have been often used separately to explore sex determination in vertebrates $[16,30,31]$, we choose to combine these two approaches in goldfish because of the significant female-to-male sex reversal induced by temperature [25] that would have prevented a clear identification of the sex determining region using only a pooled strategy, which mixes genetic $\mathrm{XY}$ males and $\mathrm{XX}$ males resulting from the sex reversal of genetic females. Because RAD-sequencing keeps track of each individual, we were able to identify sexreversed individuals in goldfish that might have masked sex-linked markers in Pool-seq.

Sex markers identification is an important step to characterize SD systems [32-38]. Using two complementary whole-genome approaches, we characterized genomic regions containing sex-linked markers. In our experimental goldfish population, these sex-linked markers are genomic DNA variations including gaps, indels and SNPs that present heterozygote polymorphisms in all males and complete homozygosity in all females. This male-specific heterozygosity pattern agrees with a male heterogametic $\mathrm{XX} / \mathrm{XY}$ system as previously reported using progeny testing of hormonally sex-reversed breeders [24]. We found, however, a strong environmental influence leading to a relatively high proportion (around 50\%) of female-to-male sex-reversal in the first experimental population that we used for the RAD-Sequencing approach. These animals were actually two-year old goldfish raised in an outdoor experimental facility and obtained at different spawning times i.e., from May-June to late September. Some of these animals experienced early development during summer time at potentially higher temperature and others had their early developmental period at lower temperatures. Considering the known effects of high temperature on female-to-male sex reversal in goldfish [25], the fact that some of these fish were exposed to a high summer temperature could explain this relatively high percentage of female-to-male sex-reversed animals. This high percentage was not found in our other experiments in which fish were raised in indoor recirculating system facilities with a tightly controlled low temperature $\left(18^{\circ} \mathrm{C}\right)$ maintained throughout the whole early development phase (3 months). This situation indeed confirms earlier findings showing that temperature is probably a major trigger of neomasculinization in goldfish, but we also found that even at this low temperature there was still a small percentage of female-to-male sex-reversal (7.8\%), suggesting that other environmental factors, potentially social factors as demonstrated in other species $[8,39]$, could also play a role on goldfish sex determination. Apart from goldfish, sex determination in other teleost fish, including Tilapia 

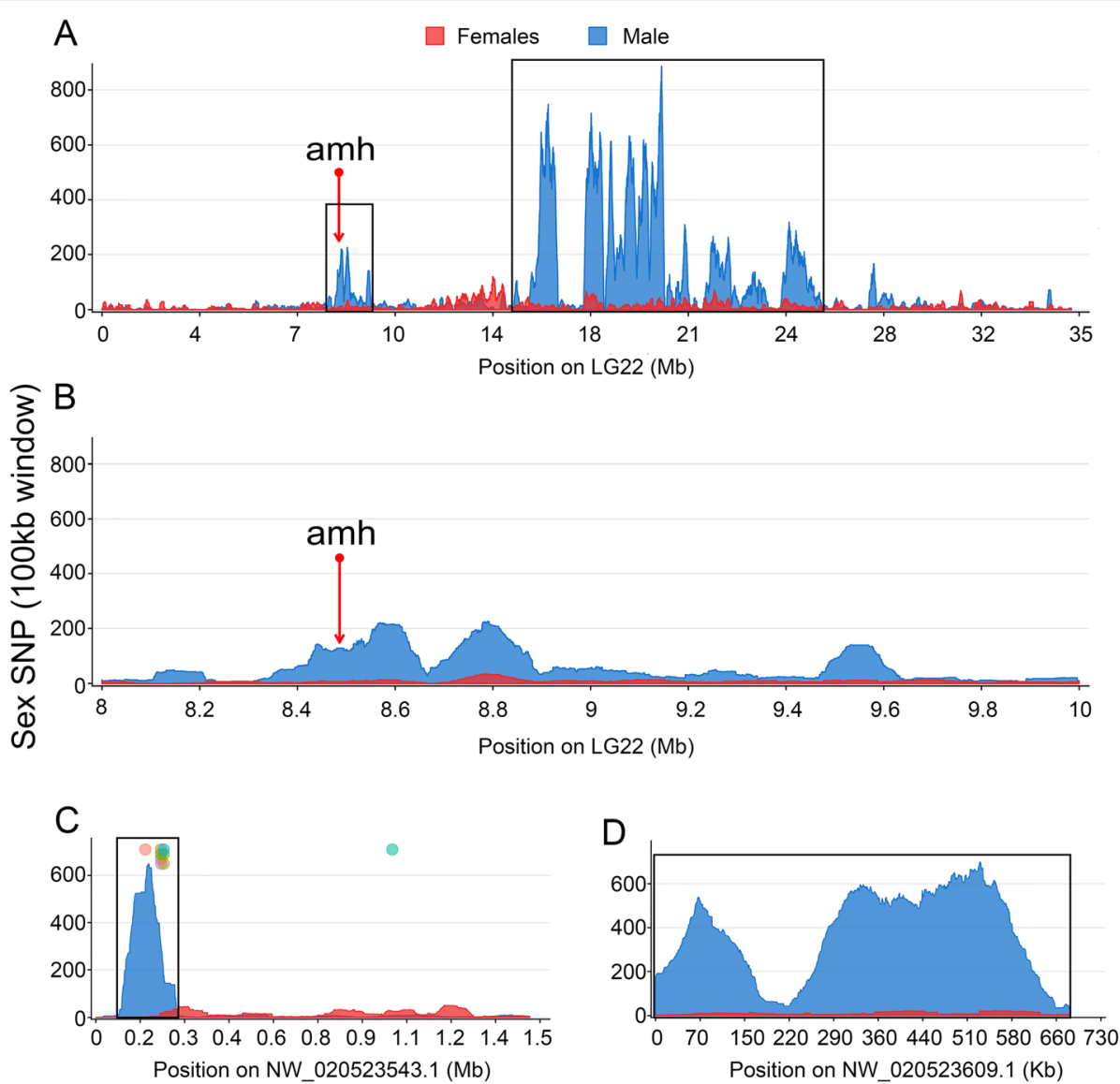

Fig. 3 Distribution of male-specific SNPs on LG22 and unplaced scaffolds NW_020523543.1 and NW_020523609.1. SNPs were counted using 100 $\mathrm{kb}$ sliding window with an output point every $500 \mathrm{bp}$ and female- and male-specific SNPs were respectively indicated by red and blue color. a A large sex-determination region was identified on LG22, which is highlighted with a black box. The candidate sex-determining gene amh is located on this LG22, but not in the high density, male-specific SNP region. The region from $8 \mathrm{Mb}$ to $10 \mathrm{Mb}$ containing amh is zoomed in panel (b). c The National Center for Biotechnology Information (NCBI) Accession number NW_020523543.1 unplaced scaffold exhibits a region around $0.1 \mathrm{Mb}$ harboring a small region (200 kb) with a high-density of male-specific SNPs. Meanwhile, sequence comparisons demonstrate that 7 malebiased RAD-tags (colored circles) on a total of 32 map with a high identity onto this scaffold. In contrast, few female-specific SNPs were enriched on this scaffold (red area). d The NCBI Accession number NW_020523609.1 unplaced scaffold is enriched in male-specific SNPs

[40], medaka [41] and tongue sole [42] is also regulated by temperature, which overrides the genetic sex determination mechanisms and leads to female-to-male sex reversal. By developing genetic sexing tools in our goldfish population allowing the identification of Y-allele carrying animals, we also brought additional evidence that some of these phenotypic males were indeed sex-reversed XX genetic females. These genetic sexing tools are indeed important for better deciphering genetic and environmental sex determination. But these genetic sexing tools have only been validated in our experimental strain of goldfish, and more work would now be needed in order to extend these results to all populations of goldfish. This is especially important in the case of goldfish as this species has a long history of human domestication and selection that could have favored switches in its sex determination system like what has been found for instance in zebrafish [27].
Sex determination in vertebrates is highly variable with the major exceptions of Eutherian mammals and birds in which $\mathrm{XX} / \mathrm{XY}$ and $\mathrm{ZZ} / \mathrm{ZW}$ monofactorial sex determination systems have been conserved over a long evolutionary period $[43,44]$. In contrast, fish exhibit much more diverse and dynamic sex determination $[9,10,45]$, with monofactorial and polyfactorial $[46,47]$ genetic systems and frequent switches and turnovers of master sexdetermining genes $[12,14,15,17,21,48]$. In our experimental goldfish population, we identified male-specific markers and obvious male-specific SNPs strongly enriched on LG22. This result confirms that goldfish has an XX-XY system [24] and also indicated that LG22 is the sex chromosome in our experimental goldfish population. Evidence is accumulating for the hypothesis that sex chromosomes, in most cases, evolve from autosomes with de novo initial evolution of a new sex determination 
mechanism that subsequently becomes fixed and extended by the suppression of recombination on the sex chromosome in the vicinity of the initial sex locus, which may increase the size of this non recombining sex determination locus [49]. In our goldfish experimental population, 11.7 Mb of LG22 contains numerous male-specific SNPs. A similar large size of the non-recombining region on the sex chromosomes was also found in tilapia including 17.9 $\mathrm{Mb}$ in Sarotherodon melanotheron (Rüppell, 1852) and 10.7 Mb in Oreochromis niloticus (Linnaeus, 1758) [30, 31]. This large non-recombining region on LG22 contains 373 gene models based on the goldfish genome annotation and also a large number of transposable elements (TEs) that were found in the male specific contigs identified by our RAD-Sex and our draft genome analysis. Enrichment of TEs around sex loci has been found in other vertebrate species [50] and may play a crucial role for suppression of recombination leading to an expansion of sex chromosome divergence.

With LG22 being the potential sex chromosome in our goldfish experimental population, it is reasonable to believe that the non-recombining region that we characterize on LG22 contains its master sex determining gene. But the only "usual suspect" master sex determining candidate found in this region and the additional nonassembled scaffolds containing sex-linked markers is the anti-Mullerian hormone gene $(a m h)$ that is located at the beginning of the LG22 non-recombining region. Duplications of amh have been characterized as the master sex determining gene in different fish species [14, 15], making Amh and members of the TGF-beta pathway $[17,19,20]$ likely candidates for this sex-determining function. But we have not been able to validate sex-linked variation neither in the amh coding DNA sequence nor in its $5 \mathrm{~kb}$ proximal promoter sequence. Even if we cannot rule out the hypothesis that amh regulation could be affected by sexspecific cis-regulatory elements located very far upstream from amh, our results do not provide any clear and direct evidence that this gene is the goldfish master sex determining gene. Indeed, not all master sex determining genes are classical "usual suspects" known to be involved in the sex-differentiation pathway like TGF-beta members [17, 19, 51], Sox3 [21], or Dmrt1 [48, 52]. For instance, the rainbow trout master sex determining gene arose from the duplication / transposition / evolution of an immunerelated gene [12]. This finding suggests that goldfish could also have an unusual master sex determining gene, preventing an easy and direct identification just with simple genome-wide analyses and candidate gene approaches.

The goldfish genome, like the genomes of the common carp and other species of the cyprinid subfamily cyprininae is characterized by a relatively recent whole genome duplication (WGD) that occurred approximately 14 million years ago [6]. This WGD adds an extra complexity to our search for sex-linked regions and sex determining candidate genes because some of these duplicated regions may still retain large blocks of high sequence similarity. The cyprininae genome duplication probably explains why we found an additional sex-biased signal on LG47 that stems from the duplication of the same ancestral chromosome as LG22. In addition to the cyprininae WGD, the current goldfish reference genome sequence [6] was assembled from the sequences of an $\mathrm{XX}$ gynogenetic animal, meaning that the LG22 sex chromosome sequence is an $\mathrm{X}$ chromosome sequence in which potential $\mathrm{Y}$ specific regions may be not present. We indeed produced a first draft genome sequence of an $\mathrm{XY}$ male but a higher contiguity male genome including long-read technology would be needed to better explore sex-chromosome differences and characterize potential sex-determining candidates.

\section{Conclusions}

Our results confirm that sex determination in goldfish is a complex mix of environmental and genetic factors, and that its genetic sex determination system is male heterogametic $(\mathrm{XX} / \mathrm{XY})$. We also characterized a relatively large non-recombining region $(\sim 11.7 \mathrm{Mb})$ on LG22 that is likely to be the Y chromosome of our goldfish experimental population. This large nonrecombining region on LG22 contains a single obvious candidate as a potential master sex gene, namely the anti-Mullerian hormone gene $(a m h)$. No sex-linked polymorphism, however, was detected in the goldfish amh gene and its $5 \mathrm{~kb}$ proximal promoter sequence. Our work provides the foundation required for additional studies that are now required to better characterize sex determination in goldfish and to characterize its master sex-determining gene.

\section{Methods \\ Experimental fish}

Our goldfish (Unité INRAE d'Expérimentale Ecologie Ecotoxicologie aquatique, or U3E-INRAE experimental aquaculture strain) population is an experimental aquaculture strain that has been maintained in our experimental facilities since 1996. It results from the initial mixture of two different populations, one coming from a commercial strain from the "Relot frères" fish farm (https://relot.fr/) and the other one that was obtained from a local aquarium trade store. Goldfish (U3E-INRAE experimental strain) used for RAD-seq and Pool-seq were reared outdoors and obtained from different spawning times i.e., between May-June and late September. These animals were sexed by the identification of gametes (sperm or oocytes) following gentle striping. Putative $\mathrm{XY}$ and $\mathrm{XX}$ males (U3E-INRAE experimental strain) were selected using Yallele specific primers and these two males were crossed 
with the same female to produce two goldfish populations that were incubated and reared indoor at $18^{\circ} \mathrm{C}$ during 3 months after fertilization to minimize the chance of sex reversal induced by temperature according to previous research [25]. Because these fish were reared indoor at $18{ }^{\circ} \mathrm{C}$ during their early development, their development was strongly slowed down. To compensate for this initial slow growth rate, the rearing temperature was gradually increased at 3 months-old to $24^{\circ} \mathrm{C}$ over a period of 7 days to avoid suddenly dramatic temperature variation. However, the growth rate of these two populations was still not comparable to goldfish populations reared in outdoor experimental facilities and at one-year old many of these fish were still small. To overcome this problem, we decided to sex these fish based on gonadal histology. Fish were euthanized at one-year old with a lethal dose of Tricaine (MS222) before dissection. Gonads of goldfish were fixed in Bouin's fixative solution for $24 \mathrm{~h}$ and then embedded gonads were cut serially into $7 \mu \mathrm{m}$ sections and stained with Hematoxylin to characterize ovarian or testicular features. Fin clips were stored in $90 \%$ alcohol for DNA extraction and genotyping. Statistics were applied to test for significant sex ratio differences and genotype/phenotype sexlinkage with a Chi-squared test $(p<0.05)$. A total of 309 fishes were used for all these experiments including animals sampled for RAD-seq and Pool-seq $(N=60)$ and the genotyping of the $\mathrm{XX}(N=1)$ and $\mathrm{XY}(\mathrm{N}=1)$ offspring $(N=121$ and 127). For RAD-seq and Pool-seq we used 30 males and 30 females in order to have a sufficient number of animals from each sex to be able to discriminate sexspecific markers from background polymorphism. As these 60 animals were sexed based on gamete production they were kept alive and not euthanized for this experimentation. For the genotyping of the $\mathrm{XX}(\mathrm{N}=1)$ and $\mathrm{XY}$ $(\mathrm{N}=1)$ offspring we designed our experiment in order to have a sufficient number $(>100)$ of animals in each family to get a precise estimation of the sex ratio.

\section{DNA extraction and genotyping}

For genotyping, fin clips were lysed with 5\% Chelex and $20 \mathrm{mg}$ Proteinase $\mathrm{K}$ at $55^{\circ} \mathrm{C}$ for $2 \mathrm{~h}$, and subsequently denatured by Proteinase $\mathrm{K}$ at $99^{\circ} \mathrm{C}$ for $2 \mathrm{~min}$. Supernatant containing genomic DNA (gDNA) was collected to a new tube after a brief centrifugation. Finally, DNA was diluted to half and stored at $-20^{\circ} \mathrm{C}$. For genome sequencing, gDNA was extracted with NucleoSpin Kits for Tissue (Macherey-Nagel, Duren, Germany) following the manufacturer's instructions. gDNA concentration and quality were measured with a NanoDrop ND2000 spectrophotometer (Thermo Scientific, Wilmington, DE) and a Qubit3 fluorometer (Invitrogen, Carlsbad, CA).

Primers were designed from the sequences of malebiased contigs for sex genotyping and a positive control (Table S1) based on our Illumina male genome assembly
(National Center for Biotechnology Information Accession number: WSJC000000000 [https://www.ncbi.nlm.nih.gov/ nuccore/WSJC000000000]) using Primer3 version 0.4.0 (http://primer3.ut.ee). These male-specific primers were found to share some sequence similarity with regions located in two unplaced contigs (National Center for Biotechnology Information Accession numbers: NW_020523543.1 [https://www.ncbi.nlm.nih.gov/nuccore/NW_020523543.1], NW_020525535.1 [https:/www.ncbi.nlm.nih.gov/nuccore/ NW_020525535.1]) and LG8 from the goldfish reference genome. Search for homologies using Blast shows that one primer pair is located in the guanylate-binding protein 1like gene (gbp1-like), while the two others are located in transposons with annotations corresponding to putative transposase element L1Md-A101/L1Md-A102/L1Md-A2 and Retrovirus-related Pol polyprotein LINE-1. PCRs were performed with $0.1 \mu \mathrm{M}$ of each primer, $50 \mathrm{ng}$ of gDNA adjusted at $50 \mathrm{ng} / \mu \mathrm{l}, 100 \mu \mathrm{M}$ dNTP mixture, and $1 \mu \mathrm{l}$ of $10 \times$ PCR Buffer (Sigma Aldrich) with 0.25 units of JumpStart Taq DNA Polymerase (Sigma Aldrich) in a total volume of $25 \mu$. The PCR thermal cycle procedures were: $94^{\circ} \mathrm{C}$ for 30s for denaturing, $58^{\circ} \mathrm{C}$ for 30 s for annealing and $72{ }^{\circ} \mathrm{C}$ for 30 s for extending for 35 cycles. Finally, PCR products were electrophoresed on $1.5 \%$ agarose gels.

\section{Restriction-site association sequencing (RAD-seq) and male-marker discovery}

Genomic DNA was extracted from 30 males and $30 \mathrm{fe}$ males and digest with the restriction enzyme SbfI for constructing a RAD-seq library according to standard protocols [53]. Briefly, for each sample, $1 \mu \mathrm{g}$ of DNA was digested using SbfI. Digested DNA was purified using AMPure PX magnetic beads (Beckman Coulters) and ligated to indexed P1 adapters (one index per sample) using concentrated T4 DNA ligase (NEB). Ligated DNA was purified using AMPure XP magnetic beads. Each sample was quantified using microfluorimetry (Qubit dsDNA HS assay kit, Thermofisher) and all samples were pooled in equal amount. The pool was fragmented on a Biorputor (Diagenode) and purified using a Minelute column (Qiagen). Sonicated DNA was size selected on an 1,5\% agarose cassette aiming for an insert size of $300 \mathrm{bp}$ to $500 \mathrm{bp}$. Size selected DNA was extracted from the gel using the Qiaquick gel extraction kit (Qiagen), repaired using the End-It DNA-end repair kit (Tebu Bio) and adenylated on its 3 ' ends using Klenow (exo-) (Tebu-Bio). P2 adapter was ligated using concentrated T4 DNA ligase (NEB) and $50 \mathrm{ng}$ of the ligated product was engaged in a 12 cycles PCR. After AMPure XP beads purification, the resulting library was checked on a Bioanalyzer (Agilent) using the DNA 1000 kit and quantified by qPCR using the KAPA Library quantification kit (Roche, ref. KK4824). The library was sequenced on one lane of Hiseq2500 in single read $100 \mathrm{nt}$ mode using the 
clustering and SBS v3 kit following the manufacturer's instructions.

Raw reads were demultiplexed with the program process_radtags.pl of Stacks with default settings. 135, 019,110 (79.1\%) reads were kept after this procedure. Demultiplexed reads were subsequently processed by the RADSex software version 2.0.0 (http://github.com/ RomainFeron/RadSex). The distribution of sequences between male and female were calculated with function distrib with all settings to default. This distribution of sequences was visualized with plot_sex_distribution function of radsex-vis (http://github.com/RomainFeron/ RADSex-vis) (Fig. 1a). Sequences significantly associated with sex were extracted using the function signif, which identifies sex-bias tags.

Male-biased tags were compared to the male de novo assembly with ncbi-blast+ (version: 2.6.0) setting the evalue cutoff to $1^{\mathrm{e}-20}$ to identify long, homologous malebiased contigs. Male specific PCR primers were designed from these contigs sequences (see Table S1) using Primer3 version 0.4.0 (http://primer3.ut.ee).

\section{Pooled genome sequencing (Pool-seq) and sex differentiated region identification}

Genomic DNA extracted from the fin clips of 13 phenotypic females and 13 genotypic males selected from the animals used for the RAD-Seq experiment, were used for the Pool-Seq analysis. The 13 genotypic males were genotyped using the three Y-allele PCR primers described above. Genomic DNA were pooled in equimolar ratio according to sex and Pool-seq libraries were generated using the Truseq nano DNA sample prep kit (Illumina, ref. FC-121-4001) following the manufacturer's instructions. Briefly, each pool was sonicated using a Bioruptor (Diagenode). The sonicated pools were repaired, size selected on magnetic beads aiming for a $550 \mathrm{pb}$ insert size and adenylated on their $3^{\prime}$ ends. Adenylated DNA was ligated to Illumina's specific adapters and, after purification on magnetic beads, was amplified in an 8 cycles PCR. Libraries were purified using magnetic beads, checked on a Fragment Analyzer (Agilent) using the HS NGS Fragment kit (DNF-474-33) and quantified by qPCR using the KAPA Library quantification kit (Roche, ref. KK4824). Each library was sequenced on half a lane of a rapid v2 flow cell (Illumina) in paired end 2x250nt mode.

Reads from the male and female pools were remapped to a genome sequence coming from a gynogenesis-derived female (National Center for Biotechnology Information Accession number: QPKE00000000 [https://www.ncbi. nlm.nih.gov/nuccore/QPKE00000000]) using BWA mem version 0.7.17 with default parameters. Then, BAM files were sorted and merged with Picard tools version 2.18.2 with default parameters. After that, PCR duplicates were removed with Picard tools. Reads with mapping quality less than 20 and that did not map uniquely were also removed with Samtools version 1.8. Subsequently, the two sex BAM files were used to generate a pileup file using samtools mpileup with per-base alignment quality disabled (-B). A sync file was created using popoolation mpileup2sync version 1.201 (parameters: --min-qual 20), which contains the nucleotide composition of each sex for each position in the reference. Finally, with this sync file, SNPs and coverage between the two sexes of all reference positions were overall calculated with PSASS (version 2.0.0, doi:https://doi.org/10. 5281/zenodo.2615936). We used a $100 \mathrm{~kb}$ sliding window with an output point every 500 bp to identify sex-specific SNPs enriched regions with PSASS. The PSASS parameters were as follows: minimum depth set to 10 (--min-depth 10), range of heterozygous SNP frequency for a sex-linked locus $0.5 \pm 0.2$ (--freq-het 0.5, --range-het 0.2), homologous SNP frequency for a sex-linked locus $>0.98$ (--freqhom 1, --range-hom 0.02), overlapped sliding window (--window-size 100,000, --output-resolution 500). Data visualization was implemented with an R package (http:// github.com/RomainFeron/PSASS-vis).

\section{Sequencing and de novo assembly of a goldfish male genome}

One genetic male was selected for de novo assembly using the Y-specific primers described above. Library was generated using the Truseq nano DNA sample prep kit (Illumina, ref. FC-121-4001) following the manufacturer's instructions. Briefly, DNA from a single male individual was sonicated using a Bioruptor (Diagenode). The sonicated DNA was repaired, size selected on magnetic beads aiming for a $550 \mathrm{pb}$ insert size and adenylated on its 3' ends. Adenylated DNA was ligated to Illumina's specific adapters and, after purification on magnetic beads, was amplified in an 8 cycles PCR. Library was purified using magnetic beads, checked on a Fragment Analyzer (Agilent) using the HS NGS Fragment kit (DNF-474-33) and quantified by qPCR using the KAPA Library quantification kit (Roche, ref. KK4824). The library was sequenced on one lane of a rapid v2 flow cell (Illumina) in paired end 2*250 nt mode. Illumina pairedend reads were assembled using DiscovarDeNovo (reference https://software.broadinstitute.org/software/discovar/ blog/) with standard parameters.

\section{Supplementary information}

Supplementary information accompanies this paper at https://doi.org/10. 1186/s12864-020-06959-3.

Additional file $\mathbf{1}$ Table S1. Sequences of the primers used for Y-allele genotyping in goldfish.

Additional file 2 Figure S1. Distribution of sex-biased SNPS on LG47. SNPs were counted using $100 \mathrm{~kb}$ sliding window with an output point every $500 \mathrm{bp}$. The top panel displays the profile of male-specific SNPs 
(blue area), while the bottom panel displays the profile of female-specific SNPs (red area).

Additional file 3 Figure S2: Dot plot comparison of LG22 and LG47 showing conserved synteny between these two linkage groups.

Additional file 4 Figure S3: Sex genotyping with Y-allele primers of the offspring of a putative XX neomale with a normal XX female. Genotyping was conducted with three $Y$-allele primers and one autosomal primer used as a gDNA quality control. Phenotypic sex was determined by gonadal histology and males and females are shown using red and yellow color respectively. Female-to-male sex-reversed animals $(N=7)$ are highlighted by red boxes. Hashes indicate animals with unknown phenotypic sex with undifferentiated gonads based on histology. The original, unprocessed gel images of this figure are available in additional file 5 .

Additional file $\mathbf{5}$ Figure $\mathbf{S 4}$. Sex genotyping with Y-allele primers of the offspring of a putative XY male with a normal XX female. Genotyping was conducted with three $Y$-allele primers and one autosomal primer used as a gDNA quality control. Phenotypic sex was determined by gonadal histology and males and females are shown using red and yellow color respectively. The female-to-male sex-reversed animal $(N=1)$ is highlighted by a red box. Hashes indicate animals with unknown phenotypic sex with undifferentiated gonads based on histology. The original, unprocessed gel images of this figure are available in additional file 5 .

Additional file 6 Sequences of putative $Y$-allele RAD-tags $(N=32)$ found in some males but absent from all females.

Additional file 7. Contig names (contigID) from a goldfish Illumina male genome assembly with homologies with the putative Y-allele RAD-tags.

Additional file 8. Annotation of potential $Y$ chromosome contigs found in our male goldfish draft genome assembly by sequence comparisons using blastx searches for genes by BLAST.

Additional file 9 Extraction of detailed information on the annotated genes in the goldfish sex determination regions [SDR $(N=373)$ ] extracted from the NCBI goldfish female genome assembly annotation file (accession number QPKE00000000).

Additional file 10. Original, unprocessed gel images of Fig. 1b, and supplementary figures 3 and 4 .

\section{Abbreviations}

RAD-seq: Restriction site-associated DNA sequencing; SNP: Single nucleotide polymorphism; SD: Sex determination; SDR: Sex differentiated region; MSD: Master sex determining genes

\section{Acknowledgements}

We are grateful to the Genotoul bioinformatics platform Toulouse MidiPyrenees (Bioinfo Genotoul) for providing computing and/or storage resources and to the INRAE-U3E and INRAE-LPGP experimental facilities for taking care of goldfish experiments.

\section{Authors' contributions}

All authors have read and approved the manuscript. YG and MW conceived and designed the experiments. Funding acquisition: YG, MS, JHP, LJ. Investigation: MW, QP, JG, EJ, AH, HP, SMB, YO, MS, JHP, LJ. Bioinformatics analysis: RF, CK, CC, MZ. Visualization: MW. Wrote the paper: MW, YG, MS, $J H P$.

\section{Funding}

This project was supported by funds from the "Agence Nationale de la Recherche" and the "Deutsche Forschungsgemeinschaft" (ANR/DFG, PhyloSex project, 2014-2016) to MS and YG. Montpellier Genomics (MGX) facility was supported by France Génomique National infrastructure, funded as part of "Investissement d'avenir" program managed by Agence Nationale pour la Recherche (contract ANR-10-INBS-09). This work was also supported by Grant-in-Aid for Scientific Research (19 K22426) to YO, and grants R01OD011116 and 5R01GM085318 from the USA National Institutes of Health to JHP. INRAE-U3E was supported by a PEARL INRAE 1036 U3E funding from the ANAEE-France National infrastructure.

\section{Availability of data and materials}

This Whole Genome Shotgun project has been deposited in the National Center for Biotechnology Information DDBJ/ENA/GenBank databases under the accession number WSJC000000000 [https://www.ncbi.nlm.nih.gov/ nuccore/WSJC000000000], The version described in this paper is version WSJC010000000. Genome sequencing reads of the male genome, the male and female pool-sequencing reads and the RAD-seq demultiplexed sequences have been deposited in the National Center for Biotechnology Information Sequence Read Archive (SRA) database, are publicly available under the BioProject accession number PRJNA592334. A gynogenesisderived female assembly containing two unplaced (NW_020523543.1 = https://www.ncbi.nlm.nih.gov/nuccore/NW_020523543.1/, NW_020525535.1 = https://www.ncbi.nlm.nih.gov/nuccore/NW_020525535.1) was obtained from National Center for Biotechnology Information under the accession number QPKE00000000 [https://www.ncbi.nlm.nih.gov/nuccore/ QPKE00000000].

\section{Ethics approval}

Research involving animal experimentation conformed to the principles for the use and care of laboratory animals, in compliance with French ("National Council for Animal Experimentation" of the French Ministry of Higher Education and Research and the Ministry of Food, Agriculture, and Forest) and European (European Communities Council Directive 2010/63/UE) guidelines on animal welfare. Under these French and European guidelines, tissue sampling on euthanized animals carried out in this study does not require any specific ethics approval.

\section{Consent for publication}

Not applicable.

\section{Competing interests}

The authors declare that they have no competing interests.

\section{Author details}

${ }^{1}$ State Key Laboratory of Developmental Biology of Freshwater Fish, College of Life Science, Hunan Normal University, Changsha, China. ${ }^{2}$ INRAE, LPGP, 35000 Rennes, France. ${ }^{3}$ Department of Ecology and Evolution, University of Lausanne, 1015 Lausanne, Switzerland. ${ }^{4}$ Swiss Institute of Bioinformatics, 1015 Lausanne, Switzerland. ${ }^{5}$ Plate-forme bio-informatique Genotoul, Mathématiques et Informatique Appliquées de Toulouse, INRAE, Castanet Tolosan, France. ${ }^{6}$ SIGENAE, GenPhySE, Université de Toulouse, INRAE, ENVT, Castanet Tolosan, France. ${ }^{7}$ Montpellier GenomiX (MGX), c/o Institut de Génomique Fonctionnelle, 141 rue de la Cardonille, 34094 Montpellier Cedex 05, France. ${ }^{8}$ Translational and Functional Genomics Branch, National Human Genome Research Institute, Bethesda, MD, USA. ' ${ }^{2}$ aboratory of Functional Genomics, Graduate School of Bioscience, Nagahama Institute of Bioscience and Technology, Nagahama, Shiga, Japan. ${ }^{10}$ Laboratory for Molecular and Developmental Biology, Institute for Protein Research, Osaka University, Suita, Osaka, Japan. ${ }^{11}$ Institute of Neuroscience, University of Oregon, Eugene, Oregon, USA. ${ }^{12}$ Developmental Biochemistry, Biozentrum, University of Würzburg, Würzburg, Germany. ${ }^{13}$ The Xiphophorus Genetic Stock Center, Department of Chemistry and Biochemistry, Texas State University, San Marcos, TX, USA.

Received: 8 January 2020 Accepted: 29 July 2020

Published online: 11 August 2020

\section{References}

1. Blanco AM, Sundarrajan L, Bertucci Jl, Unniappan S. Why goldfish? Merits and challenges in employing goldfish as a model organism in comparative endocrinology research. Gen Comp Endocrinol. 2018;257:13-28.

2. Popesku JT, Martyniuk CJ, Mennigen J, Xiong H, Zhang D, Xia X, Cossins AR, Trudeau VL. The goldfish (Carassius auratus) as a model for neuroendocrine signaling. Mol Cell Endocrinol. 2008;293(1-2):43-56.

3. Omori Y, Kon T. Goldfish: an old and new model system to study vertebrate development, evolution and human disease. J Biochem. 2019;165(3):209-18.

4. Ota KG, Abe G. Goldfish morphology as a model for evolutionary developmental biology. Wiley Interdiscip Rev Dev Biol. 2016;5(3):272-95.

5. Choe Y, Yu JE, Park J, Park D, Oh J-I, Kim S, Moon KH, Kang HY. Goldfish, Carassius auratus, as an infection model for studying the pathogenesis of Edwardsiella piscicida. Vet Res Commun. 2017;41(4):289-97. 
6. Chen Z, Omori Y, Koren S, Shirokiya T, Kuroda T, Miyamoto A, Wada H, Fujiyama A, Toyoda A, Zhang S. De novo assembly of the goldfish (Carassius auratus) genome and the evolution of genes after whole-genome duplication. Sci Adv. 2019;5(6):eaav0547.

7. Mohammad T, Moulick S, Mukherjee CK. Economic feasibility of goldfish (Carassius auratus Linn.) recirculating aquaculture system. Aquac Res. 2018; 49(9):2945-53.

8. Devlin $\mathrm{RH}$, Nagahama Y. Sex determination and sex differentiation in fish: an overview of genetic, physiological, and environmental influences. Aquaculture. 2002;208(3-4):191-364.

9. Pan Q, Anderson J, Bertho S, Herpin A, Wilson C, Postlethwait JH, Schartl M, Guiguen Y. Vertebrate sex-determining genes play musical chairs. C R Biol. 2016;339(7-8):258-62.

10. Herpin A, Schartl M. Plasticity of gene-regulatory networks controlling sex determination: of masters, slaves, usual suspects, newcomers, and usurpators. EMBO Rep. 2015;16(10):1260-74.

11. Matsuda M, Nagahama Y, Shinomiya A, Sato T, Matsuda C, Kobayashi T, Morrey CE, Shibata N, Asakawa S, Shimizu N. DMY is a Y-specific DMdomain gene required for male development in the medaka fish. Nature. 2002;417(6888):559.

12. Yano A, Guyomard R, Nicol B, Jouanno E, Quillet E, Klopp C, Cabau C, Bouchez O, Fostier A, Guiguen Y. An immune-related gene evolved into the master sex-determining gene in rainbow trout, Oncorhynchus mykiss. Curr Biol. 2012;22(15):1423-8.

13. Hattori RS, Murai Y, Oura M, Masuda S, Majhi SK, Sakamoto T, Fernandino Jl, Somoza GM, Yokota M, Strüssmann CA. A Y-linked anti-Müllerian hormone duplication takes over a critical role in sex determination. Proc Natl Acad Sci. 2012;109(8):2955-9.

14. Li M, Sun Y, Zhao J, Shi H, Zeng S, Ye K, Jiang D, Zhou L, Sun L, Tao W, et al. A tandem duplicate of anti-Müllerian hormone with a missense SNP on the $Y$ chromosome is essential for male sex determination in Nile Tilapia, Oreochromis niloticus. PLOS Genetics. 2015;11(11):e1005678.

15. Pan Q, Feron R, Yano A, Guyomard R, Jouanno E, Vigouroux E, Wen M, Busnel J-M, Bobe J, Concordet J-P. Identification of the master sex determining gene in northern pike (Esox lucius) reveals restricted sex chromosome differentiation. PLoS Gene. 2019;15(8):e1008013.

16. Feron R, Zahm M, Cabau C, Klopp C, Roques C, Bouchez O, Eche C, Valiere S, Donnadieu C, Haffray P, et al. Characterization of a Y-specific duplication/ insertion of the anti-Mullerian hormone type II receptor gene based on a chromosome-scale genome assembly of yellow perch, Perca flavescens. Mol Ecol Resour. 2020;20(2):531-43.

17. Kamiya T, Kai W, Tasumi S, Oka A, Matsunaga T, Mizuno N, Fujita M, Suetake $H$, Suzuki S, Hosoya S, et al. A trans-species missense SNP in Amhr2 is associated with sex determination in the tiger pufferfish, Takifugu rubripes (fugu). PLoS Genet. 2012;8(7):e1002798.

18. Rondeau EB, Messmer AM, Sanderson DS, Jantzen SG, von Schalburg KR, Minkley DR, Leong JS, Macdonald GM, Davidsen AE, Parker WA. Genomics of sablefish (Anoplopoma fimbria): expressed genes, mitochondrial phylogeny, linkage map and identification of a putative sex gene. BMC Genomics. 2013;14(1):452.

19. Myosho T, Otake H, Masuyama H, Matsuda M, Kuroki Y, Fujiyama A, Naruse K, Hamaguchi S, Sakaizumi M. Tracing the emergence of a novel sex-determining gene in medaka, Oryzias luzonensis. Genetics. 2012;191(1):163-70.

20. Reichwald K, Petzold A, Koch P, Downie Bryan R, Hartmann N, Pietsch S, Baumgart M, Chalopin D, Felder M, Bens $M$, et al. Insights into sex chromosome evolution and aging from the genome of a short-lived fish. Cell. 2015;163(6):1527-38

21. Takehana Y, Matsuda M, Myosho T, Suster ML, Kawakami K, Shin IT, Kohara Y, Kuroki Y, Toyoda A, Fujiyama A, et al. Co-option of Sox3 as the maledetermining factor on the $Y$ chromosome in the fish Oryzias dancena. Nat Commun. 2014:5:4157

22. Matsuda M, Sakaizumi M. Evolution of the sex-determining gene in the teleostean genus Oryzias. Gen Comp Endocrinol. 2016;239:80-8.

23. Ospina-Alvarez N, Piferrer F. Temperature-dependent sex determination in fish revisited: prevalence, a single sex ratio response pattern, and possible effects of climate change. PLoS One. 2008;3(7):e2837.

24. Yamamoto TO, Kajishima T. Sex hormone induction of sex reversal in the goldfish and evidence for male heterogamity (XX/XY). J Exp Zool. 1968; 168(2):215-21.

25. Goto-Kazeto R, Abe Y, Masai K, Yamaha E, Adachi S, Yamauchi K. Temperature-dependent sex differentiation in goldfish: establishing the temperature-sensitive period and effect of constant and fluctuating water temperatures. Aquaculture. 2006;254(1-4):617-24.

26. Chen Z, Omori Y, Koren S, Shirokiya T, Kuroda T, Miyamoto A, Wada H, Fujiyama A, Toyoda A, Zhang S et al: De novo assembly of the goldfish (Carassius auratus) genome and the evolution of genes after whole genome duplication. 2018

27. Anderson JL, Mari AR, Braasch I, Amores A, Hohenlohe P, Batzel P, Postlethwait JH. Multiple sex-associated regions and a putative sex chromosome in zebrafish revealed by RAD mapping and population genomics. PLoS One. 2012;7(7):e40701.

28. Gamble T. Using RAD-seq to recognize sex-specific markers and sex chromosome systems. Mol Ecol. 2016;25(10):2114-6.

29. Schlötterer $C$, Tobler $R$, Kofler $R$, Nolte $V$. Sequencing pools of individuals - mining genome-wide polymorphism data without big funding. Nat Rev Genet. 2014;15(11):749-63.

30. Gammerdinger WJ, Conte MA, Baroiller J-F, D'cotta H, Kocher TD. Comparative analysis of a sex chromosome from the blackchin tilapia, Sarotherodon melanotheron. BMC Genomics. 2016;17(1):808.

31. Gammerdinger WJ, Conte MA, Acquah EA, Roberts RB, Kocher TD. Structure and decay of a proto-Y region in Tilapia, Oreochromis niloticus. Bmc Genomics. 2014;15(1):975.

32. Kincaid-Smith J, Boissier J, Allienne JF, Oleaga A, Djuikwo-Teukeng F, Toulza E. A genome wide comparison to identify markers to differentiate the sex of larval stages of Schistosoma haematobium, Schistosoma bovis and their respective hybrids. PLoS Negl Trop Dis. 2016;10(11):e0005138.

33. Charlesworth D, Mank JE. The birds and the bees and the flowers and the trees: lessons from genetic mapping of sex determination in plants and animals. Genetics. 2010;186(1):9-31.

34. Pan ZJ, Li XY, Zhou FJ, Qiang XG, Gui JF. Identification of sex-specific markers reveals male heterogametic sex determination in Pseudobagrus ussuriensis. Mar Biotechnol (NY). 2015;17(4):441-51.

35. Carmichael SN, Bekaert M, Taggart JB, Christie HR, Bassett DI, Bron JE, Skuce PJ, Gharbi K, Skern-Mauritzen R, Sturm A. Identification of a sex-linked SNP marker in the salmon louse (Lepeophtheirus salmonis) using RAD sequencing. PLoS One. 2013;8(10):e77832.

36. Kafkas S, Khodaeiaminjan M, Guney M, Kafkas E. Identification of sex-linked SNP markers using RAD sequencing suggests ZW/ZZ sex determination in Pistacia vera L. BMC Genomics. 2015;16:98

37. Gamble T, Zarkower D. Identification of sex-specific molecular markers using restriction site-associated DNA sequencing. Mol Ecol Resour. 2014;14(5):902-13.

38. Fowler BL, Buonaccorsi VP. Genomic characterization of sex-identification markers in Sebastes carnatus and Sebastes chrysomelas rockfishes. Mol Ecol. 2016;25(10):2165-75

39. Kobayashi $Y$, Nagahama $Y$, Nakamura M. Diversity and plasticity of sex determination and differentiation in fishes. Sex Dev. 2013;7(1-3):115-25.

40. Wessels S, Hörstgen-Schwark G. Temperature dependent sex ratios in selected lines and crosses with a YY-male in Nile tilapia (Oreochromis niloticus). Aquaculture. 2011;318(1-2):79-84.

41. Hattori R, Gould R, Fujioka T, Saito T, Kurita J, Strüssmann C, Yokota M, Watanabe S. Temperature-dependent sex determination in Hd-rR medaka Oryzias latipes: gender sensitivity, thermal threshold, critical period, and DMRT1 expression profile. Sexual Development. 2007;1 (2):138-46.

42. Shao C, Li Q, Chen S, Zhang P, Lian J, Hu Q, Sun B, Jin L, Liu S, Wang Z. Epigenetic modification and inheritance in sexual reversal of fish. Genome Res. 2014;24(4):604-15.

43. Wallis $M$, Waters $P$, Graves J. Sex determination in mammals-before and after the evolution of SRY. Cell Mol Life Sci. 2008:65(20):3182.

44. Ellegren $\mathrm{H}$. Evolution of the avian sex chromosomes and their role in sex determination. Trends Ecol Evol. 2000;15(5):188-92.

45. Mank JE, Avise JC. Evolutionary diversity and turn-over of sex determination in teleost fishes. Sex Dev. 2009:3(2-3):60-7.

46. Roberts NB, Juntti SA, Coyle KP, Dumont BL, Stanley MK, Ryan AQ, Fernald $\mathrm{RD}$, Roberts RB. Polygenic sex determination in the cichlid fish Astatotilapia burtoni. BMC Genomics. 2016:17:835.

47. Liew WC, Bartfai R, Lim Z, Sreenivasan R, Siegfried KR, Orban L. Polygenic sex determination system in Zebrafish. PLoS One. 2012;7(4):e34397.

48. Cui Z, Liu Y, Wang W, Wang Q, Zhang N, Lin F, Wang N, Shao C, Dong Z, Li $Y$. Genome editing reveals dmrt1 as an essential male sex-determining gene in Chinese tongue sole (Cynoglossus semilaevis). Sci Rep. 2017;7:42213.

49. Wright $A E$, Dean $R$, Zimmer F, Mank JE. How to make a sex chromosome. Nat Commun. 2016;7:12087. 
50. Chalopin D, Volff JN, Galiana D, Anderson JL, Schartl M. Transposable elements and early evolution of sex chromosomes in fish. Chromosom Res. 2015;23(3):545-60.

51. Rondeau EB, Laurie CV, Johnson SC, Koop BF. A PCR assay detects a malespecific duplicated copy of anti-Müllerian hormone (amh) in the lingcod (Ophiodon elongatus). BMC Res Notes. 2016;9(1):230.

52. Nanda I, Kondo M, Hornung U, Asakawa S, Winkler C, Shimizu A, Shan Z, Haaf T, Shimizu N, Shima A, et al. A duplicated copy of DMRT1 in the sexdetermining region of the $Y$ chromosome of the medaka, Oryzias latipes. Proc Natl Acad Sci U S A. 2002;99(18):11778-83.

53. Baird NA, Etter PD, Atwood TS, Currey MC, Shiver AL, Lewis ZA, Selker EU, Cresko WA, Johnson EA. Rapid SNP discovery and genetic mapping using sequenced RAD markers. PLoS One. 2008;3(10):e3376.

\section{Publisher's Note}

Springer Nature remains neutral with regard to jurisdictional claims in published maps and institutional affiliations.

Ready to submit your research? Choose BMC and benefit from:

- fast, convenient online submission

- thorough peer review by experienced researchers in your field

- rapid publication on acceptance

- support for research data, including large and complex data types

- gold Open Access which fosters wider collaboration and increased citations

- maximum visibility for your research: over $100 \mathrm{M}$ website views per year

At BMC, research is always in progress.

Learn more biomedcentral.com/submissions 